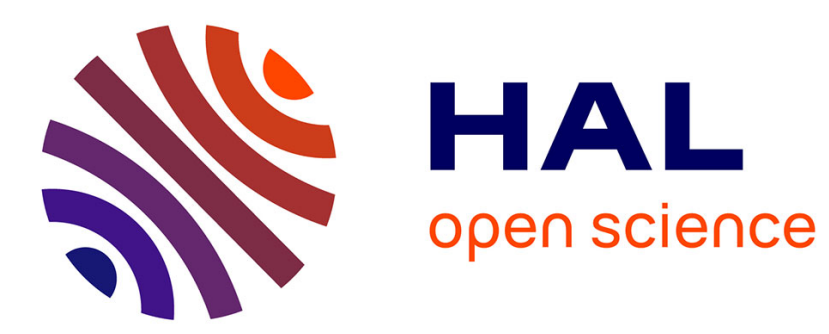

\title{
Life Cycle of Products and Cycles
}

\author{
Jean de Beir, Mouez Fodha, Francesco Magris
}

\section{To cite this version:}

Jean de Beir, Mouez Fodha, Francesco Magris. Life Cycle of Products and Cycles. 2008. halshs00348862

\section{HAL Id: halshs-00348862 \\ https://shs.hal.science/halshs-00348862}

Submitted on 22 Dec 2008

HAL is a multi-disciplinary open access archive for the deposit and dissemination of scientific research documents, whether they are published or not. The documents may come from teaching and research institutions in France or abroad, or from public or private research centers.
L'archive ouverte pluridisciplinaire HAL, est destinée au dépôt et à la diffusion de documents scientifiques de niveau recherche, publiés ou non, émanant des établissements d'enseignement et de recherche français ou étrangers, des laboratoires publics ou privés. 


\section{Documents de Travail du Centre d'Economie de la Sorbonne}

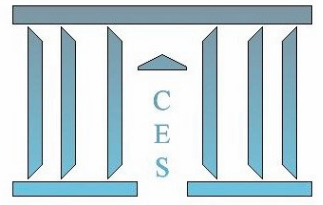

Life Cycle of Products and Cycles

Jean DE BEIR, Mouez FodHA, Francesco MAGRIS

2008.91 


\title{
Life Cycle of Products and Cycles
}

\author{
JEAN DE BEIR \\ EPEE, University Evry-Val-d'Essonne.
}

Bd. F. Mitterrand, 91025 Evry Cedex, France.

Tel.: +33 (0) 169477060 .

(jean.debeir@univ-evry.fr)

Mouez Fodha

Centre d'Economie de la Sorbonne and Paris School of Economics.

University Panthéon-Sorbonne.

Maison des Sciences Economiques, 106-112 Bld de l'Hôpital, 75013 Paris Cedex, France.

Tel.: +33 (0) 1440782 21. Fax.: +33 (0) 144078231.

(fodha@univ-paris1.fr)

FRANCESCO MAGRIS

EPEE, University Evry-Val d'Essonne.

Bd. F. Mitterrand, 91025 Evry Cedex, France.

(francesco.magris@univ-evry.fr)

May 2008 
Life Cycle of Products and Cycles

Corresponding Author:

Mouez Fodha

Address: Centre d'Economie de la Sorbonne, University Paris 1 Panthéon-Sorbonne. Maison des Sciences Economiques, 106-112 Bld de l'Hôpital, 75013 Paris Cedex, France.

Telephone: +33 (0) 144078221 .

Fax: +33(0) 144078231 .

Mailing address: fodha@univ-paris1.fr 


\begin{abstract}
The aim of this paper is to examine whether the development of waste recycling activities can be a source of economic fluctuations. We assume that the recycling sector has four fundamental characteristics. (i) The production factors are restricted by the production of the last period. (ii) These production factors are waste for which the price determination is non-competitive. (iii) It produces a recycled good, which is a perfect substitute to the primary good. (iv) It reduces waste stream. We consider the simplest economy with an infinitely lived agent and a life cycle hypothesis for the goods. We show that the equilibrium is unique and is always determinate. In spite of the lack of indeterminacy, however, our system can display cyclical behavior, depending on some usual conditions on parameters. Namely, the steady-state may undergo a Flip and a Hopf bifurcation.
\end{abstract}

JEL classification: E32; Q53.

Keywords: cycles; recycling; waste. 


\section{Introduction}

Waste recycling activity is an instrument for a sustainable development policy. Therefore, the European Union had adopted in 2002 a Directive concerning the recovery and the recycling of electrical and electronic waste products. The adoption of this Directive onto national law statutes has reinforced financial subsidies aimed at the recovery and recycling of the diverse constituent waste of these goods at the end of their useful life. ${ }^{1}$ In addition to environmental concerns, waste recycling activity corresponds to an industrial reality and to technological constraints. In France, for example, more than $58 \%$ of the paper industry's input is from recycled paper and cardboard. The recovery rate ${ }^{2}$ is as much as $54 \%{ }^{3}$ Recycling is a growing industry for a number of raw materials. Some European countries, for example, achieve record numbers in the recycling of glass (96\% in Switzerland, $92 \%$ in Sweden, $88 \%$ in Germany and in Belgium, but only $58 \%$ in France in 2003). ${ }^{4}$

The recycling sector has four fundamental characteristics. First, the factors of production are restricted by the production of preceding periods. Secondly, these factors are waste for which the price determination is non-competitive. ${ }^{5}$ Third, it produces a recycled good, which is a perfect substitute to the primary good. Finally, it reduces waste stream.

Economic literature analyses recycling from three theoretical points of view. First, recycling is integrated into the analysis of industrial organization; following the Alcoa affair in 1945, studies have measured the possible erosion of monopoly power by the competitive activity applied by manufacturers of recycled products. They showed that Alcoa's control of raw aluminium production allowed it to conserve its market power when faced by a competitive fringe of recyclers. As a matter of fact, the growth of the aluminium market was to the advantage of the monopoly: it was able to maintain its rent through controlling its raw material (Gaskins; 1974, Swan; 1980). Furthermore, in the period between the two World Wars, there were technical problems in the recovery and recycling of aluminium which hampered secondary production and reduced the possibilities of substitutability of primary and recycled products (Suslow; 1986, Grant; 1999). ${ }^{6}$

Secondly, the economic interest of natural resources allows us to understand the usefulness of recycling when faced with the diminution of availability of resources in the future; recycling postpones the working-out of mining resources and reduces the intensity of forestry exploitation (Mäler; 1974, Dasgupta and Heal; 1979).

Thirdly, environmental economics analyses recycling as an instrument to reduce negative 
externalities. The question of recycling is introduced into this research via the problem of domestic waste (Lusky; 1976). Recovery and recycling are methods of treating waste which complement other processes (Keeler and Renkow; 1994). Extended research into industrial pollution and into the consumer waste was carried out by Palmer and Walls (1997) within a partial equilibrium framework. Fullerton and Wu (1998) present a general equilibrium model in which producers offer a good using raw and recycled materials, while choosing their level of packaging and recycling. The level of waste has an influence on the welfare of consumers and these studies show that it is possible to introduce a cost incentive measure (Fullerton and Kinnaman; 1995, Choe and Fraser; 1999). These general or partial equilibrium models analyze economic instruments (rarely command and control instruments) aimed at reducing negative externalities arising from the disposal of household waste, of illicit household behavior in terms of waste and the rewarding of effort for households sorting their waste.

This article takes into account the four characteristics of the recycling sector highlighted by these studies, but we consider these characteristics simultaneously in a general equilibrium framework. More precisely, we assume the following properties: (i) the quantity of inputs recovered by this sector is limited by virgin goods produced in the preceding period; (ii) waste prices are non competitive; (iii) primary and secondary goods are perfect substitute; (iv) recycling is an instrument for reducing waste linked to industrial production.

The aim of this paper is to examine whether the development of waste recycling activities can be a source of economic fluctuations. We consider a recovery and recycling public sector in a dynamic general equilibrium framework for the simplest economy i.e. without externalities and no waste market. The framework is a Ramsey-Cass-Koopmans model with infinitely lived agents with size normalized to one and a life cycle hypothesis for the goods (two periods of use).

We show that, in this very simple economy, in spite of the lack of indeterminacy, however, our system can display cyclical behavior, depending on some weak conditions on the propensity to consumption relative to that to investment and on three elasticities: the recycling production function elasticity, the elasticity of the interest rate and the elasticity of marginal utility. Namely, the steady state may undergo a flip and a Hopf bifurcation, with the associated two-period cycles and closed orbits arising near the (unique) steady state, respectively. This result is in contrast with the standard one-sector models with inelastic labor supply, which, as shown in Boldrin and Rustichini (1994), do not display neither indeterminacy nor cyclical behavior. On the other hand, our results confirm those of Seegmuller and Verchère (2007) which show that, in a context 
of overlapping generations, the account for environment can be a source of cyclical behavior and, in their specific case, endogenous fluctuations.

We finally argue that, if recovery - recycling activity is defined by standards policy, government may choose the level of the recovery or/and recycling rate such as cycles are avoided.

The paper is organized as follows. Section 2 presents the model and characterizes the competitive equilibrium. Section 3 analyses the stability conditions and determines the flip and Hopf bifurcations. The last section concludes.

\section{The model}

\subsection{Households}

Assume population is normalized to one, there is no growth in its size and individuals are all identical. The representative infinite-lived household seeks to maximize overall utility, as given by:

$$
\operatorname{Max} \sum_{t=1}^{\infty} \beta^{t} u\left(c_{t}\right)
$$

where $c_{t}$ is consumption at time $t$ and $\beta \in[0,1]$ is the constant discount factor. The individual's resource constraint is:

$$
c_{t}+k_{t+1}=w_{t}+\left(1-\delta+r_{t}\right) k_{t}+\pi_{t}
$$

where $w_{t}$ is the wage, $r_{t}$ is the interest rate paid on saving, $\delta \in[0,1]$ is the depreciation rate of capital, $k_{t}$ represents capital stock and $\pi_{t}$ measures the profit of the representative firm operating in the recycling sector, which we will discuss later on. The first order conditions yield to the Euler equation

$$
u^{\prime}\left(c_{t}\right)=\beta\left(1-\delta+r_{t+1}\right) u^{\prime}\left(c_{t+1}\right)
$$

This relation determines the optimal consumption path of the representative household.

\section{$2.2 \quad$ Virgin sector}

Virgin production satisfies the usual assumptions. The virgin production sector consists of one competitive representative firm, characterized by a production function $f($.$) which has constant$ returns to scale and satisfies the Inada conditions. Production is a function of capital. The firm's profit $\pi_{t}^{V}$ writes:

$$
\pi_{t}^{V}=f\left(k_{t}\right)-r_{t} k_{t}-w_{t}
$$


Since markets are competitive, capital and labor earn their marginal products, i.e. $r_{t}=f^{\prime}\left(k_{t}\right)$ and $w_{t}=f\left(k_{t}\right)-f^{\prime}\left(k_{t}\right) k_{t}$.

\subsection{Recycled public production}

A public recycling sector competes directly with the virgin production sector. Its output is $y^{R}$. Secondary production and virgin production are perfect substitutes. In this sector, the public firm includes recovery and recycling activities. As Swan (1980) and Martin (1982) stated, we suppose that the unique production factor is the recovered used virgin goods and that the returns are decreasing (a characteristic of the technology of recovery). The program of the recovery-recycling sector is:

$$
\left\{\begin{array}{c}
\pi_{t}=g\left(R_{t}\right)-p R_{t} \\
\text { w.r.t. } \quad R_{t}=\phi y_{t-1}^{V}
\end{array}\right.
$$

where $R_{t}$ is the quantity of scrap purchased. The recycling function $y_{t}^{R}=g\left(R_{t}\right)$ satisfies the Inada conditions. $g($.$) is strictly concave. The constraint shows that the virgin good lasts for$ one period before it is recycled; i.e. period t's virgin production is recovered in period $t+1$ or it is definitely lost. Consequently, the stock of available waste in $t$ depends on the amount of the virgin goods produced during the preceding period $t-1$ : the quantity of waste available for recovery in $t$ is supposed equal to $\phi y_{t-1}^{V}$. By definition, $\phi \in[0,1[$ i.e. a constant share of the virgin goods is lost each period at the scrap recovery stage (depreciation cost).

The waste is collected and supplied to the recovery-recycling public sector by the government at the price $p$. This price is exogenous and set arbitrarily by the government. ${ }^{7} p$ measures also some costs linked to the recycling activity: a share is lost in the secondary production (shrinkage cost). ${ }^{8}$

Assume that there is no profit maximization task. This assumption corresponds to the three main characteristics of this sector.

(i) The recycling rate $\phi$ is defined by technical standards for some types of waste. In order to comply with the objectives of the European Directive (2004/12/EC) on packaging and packaging waste, Member States shall take the necessary measures to attain the following target, among others, covering the whole of their territory: no later than 31 December 2008 between $55 \%$ as a minimum and $80 \%$ as a maximum by weight of packaging will be recycled.

(ii) Moreover, local government control recovers the recyclable material in a waste stream, 
respecting preference order defined by EC environmental policy. It corresponds to a goal ranking between recycling, energy recovery and landfilling.

(iii) Finally, it exists in European countries, private companies accredited by the public authorities (for example DSD in Germany) which have to sort all the available households waste packaging.

Then, the recovery - recycling sector profit is:

$$
\pi_{t}^{R}=g\left(\phi f\left(k_{t-1}\right)\right)-A f\left(k_{t-1}\right)
$$

with $A=p \phi$. Hence, there is recycling activity at time $t$ if and only if $\pi_{t} \geqslant 0$, implying:

$$
\frac{g\left(\phi f\left(k_{t-1}\right)\right)}{f\left(k_{t-1}\right)} \geq A
$$

We assume that there exists $\bar{p}$ such that $\bar{p}=\frac{g(\phi f)}{\phi f}>p \geq 0$.

\section{Intertemporal equilibrium and steady state}

Consider (2) to be verified at the steady state of the model and nearby, and (1) to represent profits in the recycled goods production sector. Then, our dynamic system includes in each period the variables $c_{t}, c_{t+1}, k_{t-1}, k_{t}, k_{t+1}$. Namely, the first formula defines the Euler equation, while the second the equilibrium in goods market. Therefore we have the following definition.

Definition 1 An intertemporal equilibrium of the economy is a sequence $\left\{c_{t}, c_{t+1}, k_{t-1}, k_{t}, k_{t+1}\right\}_{t=0}^{\infty}$ satisfying the following dynamic system:

$$
\mid \begin{aligned}
& u^{\prime}\left(c_{t}\right)=\beta\left(1-\delta+f^{\prime}\left(k_{t+1}\right)\right) u^{\prime}\left(c_{t+1}\right) \\
& c_{t}+k_{t+1}=f\left(k_{t}\right)+(1-\delta) k_{t}+g\left(\phi f\left(k_{t-1}\right)\right)-A f\left(k_{t-1}\right)
\end{aligned}
$$

together with the initial conditions $k_{0}, k_{-1}$ and the usual transversality one.

One immediately obtains that the steady state of system (3) is unique and defined by:

$$
\mid \begin{aligned}
& \beta\left(1-\delta+f^{\prime}(k)\right)=1 \Longrightarrow f^{\prime}(k)=\frac{1}{\beta}-(1-\delta) \equiv \theta>0 \\
& c=f(k)+(1-\delta) k+g(\phi f(k))-A f(k)-k
\end{aligned}
$$

Fore sake of simplicity in notation, we will not label the steady state values of capital and consumption, but when they are not indexed by the time it is supposed that we are referring to the stationary values of the variables under study. 
Remark 1 System (3) is a second-order difference equation which can be expressed, as we will see below, as a three-dimensional first-order difference system. Notice that (3) is not optimal since when maximizing, agents do not take into account the possibility of recycling previous-period output. However, the study of the optimal dynamics is quite difficult since it involves the sequence of the variables $\left\{c_{t}, c_{t+1}, c_{+2}, k_{t-1}, k_{t}, k_{t+1}\right\}_{t=0}^{\infty}$. Indeed, in such a case, the Euler equation would include three consecutive marginal utilities, since a decrease of a unit of consumption in period $t$ allows an increase in consumption in the next period, by investing it, and also in period $t+2$, by means of the recycling sector. It follows that we would have to study a four-dimensional firstorder difference equation, which turns out to be hard to handle. Nevertheless, we will present some optimal results and give intuitions in section 6 .

\section{Stability analysis}

Setting $z_{t} \equiv k_{t-1}$ we can rewrite system (3) in the following way:

$$
\left\{\begin{array}{l}
k_{t+1}=f\left(k_{t}\right)+(1-\delta) k_{t}-c_{t}+g\left(\phi f\left(z_{t}\right)\right)-A f\left(z_{t}\right) \\
u^{\prime}\left(c_{t}\right)=\beta\left(1-\delta+f^{\prime}\left(k_{t+1}\right)\right) u^{\prime}\left(c_{t+1}\right) \\
z_{t+1}=k_{t}
\end{array}\right.
$$

Then, (5) is a first-order three-dimensional system. In order to study the stability properties of such a system, we linearize it around its stationary solution. Notice that the system included two predetermined variables, $k_{t-1}$ and $k_{t}$ and one which the agents must choose, $c_{t}$. It follows that in order to be indeterminate, the Jacobian must include three stable eigenvalues; in the opposite case it will be determinate, either a saddle (one or two unstable eigenvalues) or a source (three unstable eigenvalues). If we linearize system (5) around its stationary solution and we assume that there are not eigenvalues lying on the unitary circle, we get the following Jacobian matrix:

$$
J=\left[\begin{array}{ccc}
\frac{1}{\beta} & -1 & B \\
-\theta \frac{c}{k} \frac{\varepsilon_{r}}{\varepsilon_{c}} & \beta \theta \frac{c}{k} \frac{\varepsilon_{r}}{\varepsilon_{c}}+1 & -\beta \theta \frac{c}{k} \frac{\varepsilon_{r}}{\varepsilon_{c}} B \\
1 & 0 & 0
\end{array}\right]
$$

where $\varepsilon_{g} \equiv \frac{g^{\prime}(\phi f(k)) \phi f(k)}{g(\phi f(k))}$ is the recycling production function elasticity, $s \equiv \frac{f^{\prime}(k) k}{f(k)}$ is the gross capital share in the virgin production, $\varepsilon_{r}=\left|\frac{\frac{d r}{r}}{\frac{d k}{k}}\right|=-\frac{f^{\prime \prime}(k) k}{f^{\prime}(k)}>0$ the elasticity of the interest rate, $\varepsilon_{c}=-\frac{u^{\prime \prime}(c) c}{u^{\prime}(c)}>0$ the elasticity of marginal utility (i.e. inverse of the elasticity of intertemporal substitution in consumption) and $B \equiv \varepsilon_{g} s\left(\frac{c}{k}+\delta+(A-1) \frac{\theta}{s}\right)-A \theta$. The associated 
characteristic polynomial is:

$$
P(\lambda)=\lambda^{3}-\left(1+\frac{1}{\beta}+\frac{c}{k} \theta \frac{\varepsilon_{r}}{\varepsilon_{c}} \beta\right) \lambda^{2}+\left(\frac{1}{\beta}-B\right) \lambda+B
$$

In order to study the stability of system (5) we evaluate the characteristic polynomial at, respectively, $-1,0,1$ which gives $P(-1)=-\left(2+\frac{2}{\beta}+\frac{c}{k} \theta \frac{\varepsilon_{r}}{\varepsilon_{c}} \beta\right)+2 B, P(0)=B$ and $P(1)=$ $-\frac{c}{k} \theta \frac{\varepsilon_{r}}{\varepsilon_{c}} \beta<0$. Since $P(1)$ is always negative and $\lim _{\lambda \rightarrow+\infty} P(\lambda)=+\infty$ one immediately verifies that there always exists a real eigenvalue greater than one, as it is stated in the following Proposition. Actually, this follows from the fact that the steady state is unique, properties that generically rules out the existence of saddle node bifurcations.

Proposition 2 The Jacobian $J$ possesses a real eigenvalue greater than one.

Since our system has two predefined variables, $k_{t-1}$ and $k_{t}$, the existence of an unstable eigenvalue implies that system (5) is always determinate.

Corollary 3 System (5) is always determinate. It follows that it can be a saddle or a sink, according to the number of stable eigenvalues.

Remark 2 Let us observe that although $J$ has at least a real positive eigenvalue (namely greater than one), however the sign of the other two crucially depends on the sign and size of $B$. Specifically, if $P(-1)$ and $P(0)$ are negative, all three eigenvalues could be positive. Therefore, the convergence towards the steady-state will be monotonic. If $P(-1)$ is positive and $P(0)$ negative, there could be two negative eigenvalues with one of them lower than minus one and the other included in $(-1,0)$, in the case that $P(\lambda)$ was not strictly increasing in $[0, \infty)$. In such a case, the system will move towards its steady-state following an oscillatory path.

In spite of the lack of indeterminacy, however, system (5) can display cyclical behavior; namely, as we will show, the steady state may undergo a flip and a Hopf bifurcation. The impossibility of getting instead a saddle node bifurcation, as we have already said, is due to the uniqueness of the steady state. We will carry out our bifurcation analysis with respect to perturbation in the elasticity $\varepsilon_{g} \in[0,1]$ of the function $g$.

\subsection{Flip bifurcation}

A flip bifurcation occurs when one eigenvalue goes through -1 . Since the roots of the characteristic polynomial correspond to the eigenvalues, the condition boils down to find a value of 
$\varepsilon_{g}$ included between zero and one and solving $P(-1)=0$. Straightforward computation shows that $P(-1)=0$ if and only if $-\left(2+\frac{2}{\beta}+\frac{c}{k} \theta \frac{\varepsilon_{r}}{\varepsilon_{c}} \beta\right)+2 B=0$. Therefore the bifurcation value for $\varepsilon_{g}$ is

$$
\varepsilon_{g}^{F}=\frac{1+\frac{1}{\beta}+\frac{c}{k} \theta \frac{\varepsilon_{r}}{\varepsilon_{c}} \frac{\beta}{2}+A \theta}{s\left(\frac{c}{k}+\delta+(A-1) \frac{\theta}{s}\right)}
$$

Since $\varepsilon_{g}$ must belong to $[0,1]$ in order to get a flip bifurcation the Right Hand Side (RHS) of (7) must be included in this interval; the previous inequality is respected when

$$
\frac{1+\frac{1}{\beta}-s \delta+\theta}{s-\theta \frac{\varepsilon_{r}}{\varepsilon_{c}} \frac{\beta}{2}}<\frac{c}{k}
$$

under the conditions that $A-1+\left(\frac{c}{k}+\delta\right) \frac{s}{\theta}>0$ and $s-\theta \frac{\varepsilon_{r}}{\varepsilon_{c}} \frac{\beta}{2}>0$. Condition (8) is more likely to be respected as soon as either the elasticity of intertemporal substitution in consumption is high or the elasticity of the interest rate is low. In the limit case $\varepsilon_{r}=0$ or $\varepsilon_{c}=+\infty$, we have that a flip bifurcation does exist when

$$
\frac{1}{s}+\frac{1}{\beta s}-\delta+\theta / s<\frac{c}{k}
$$

In other words, economies characterized by a higher propensity to consume are more likely to display a flip bifurcation and therefore, as showed among the others in Grandmont (1988), two-period cycles. All the previous results are summarized in the following Proposition.

Proposition 4 Under condition (8) the steady state of system (5) undergoes a flip bifurcation for the elasticity of the recycling production function defined in (7). Then, there will exist near the state a stable or unstable two-period cycle, according to the direction of the bifurcation.

We now provide an explication of the mechanism at the base of the occurrence of a two-period cycle. Let fix a given level of $k$, say $k_{1}$. If it is relatively high and the propensity to consume is high too, in the next period the stock of capital will be lower, say $k_{2}<k_{1}$. If we are in the region of the recycling production function in which the profits are decreasing, in the following period total production will be higher, and so investment will increase by attaining again the level of capital $k_{1}$. A similar argument does hold in the case in which we are in the region of the recycling production function in which profits are increasing. The mechanism described explain why, in order to have cycles, the propensity to consume must be large enough. 


\subsection{The Hopf-Poincaré bifurcation}

In order to verify if system (5) displays a Hopf bifurcation, we must check if there exists a configuration such that two complex eigenvalues have modulus equal to one. If this is true, it follows that there will exist around the steady state a closed curve, attracting or repulsing, according to the direction of the bifurcation (Grandmont; 1988). As it is well known, the characteristic polynomial can be written as

$$
P(\lambda)=\lambda^{3}-T \lambda^{2}+\Sigma \lambda-D
$$

where $T$ is the Trace, $\Sigma$ the Sum of Minors of order two and $D$ the Determinant. If there exists a Hopf bifurcation, the Determinant must be a real root of the polynomial, which should be written as

$$
(\lambda-D)\left(\lambda^{2}-2 a \lambda+1\right)
$$

for some $a$ such that $|a|<1$. In fact, in such a configuration, $a$ is the common real part of the two eigenvalues with unitary modulus. In the Appendix, we show that the bifurcation value for the elasticity of the recycling production function is the following:

$$
\varepsilon_{g}^{H}=\frac{-\left(\frac{c}{k} \theta \frac{\varepsilon_{r}}{\varepsilon_{c}} \beta+\frac{1}{\beta}\right)-\sqrt{\left(\frac{c}{k} \theta \frac{\varepsilon_{r}}{\varepsilon_{c}} \beta\right)^{2}+2 \frac{c}{k} \theta \frac{\varepsilon_{r}}{\varepsilon_{c}}+\left(\frac{1}{\beta}-2\right)^{2}}+2 A \theta}{2 s\left(\frac{c}{k}+\delta+(A-1) \frac{\theta}{s}\right)}
$$

Now, we must prove that $\varepsilon_{g}^{H}$ is included between 0 and 1 . Since the denominator is positive, there will exist a value for $A$ large enough such that $\varepsilon_{g}^{H}>0$ is verified. Such $A$ is given by

$$
A>\frac{\left(\frac{c}{k} \theta \frac{\varepsilon_{r}}{\varepsilon_{c}} \beta+\frac{1}{\beta}\right)+\sqrt{\left(\frac{c}{k} \theta \frac{\varepsilon_{r}}{\varepsilon_{c}} \beta\right)^{2}+2 \frac{c}{k} \theta \frac{\varepsilon_{r}}{\varepsilon_{c}}+\left(\frac{1}{\beta}-2\right)^{2}}}{2 \theta}
$$

Condition $\varepsilon_{g}^{H}<1$ does not depend upon $A$. Actually, to ensure this last inequality, we obtain the following condition:

$$
\frac{-\left(\frac{c}{k} \theta \frac{\varepsilon_{r}}{\varepsilon_{c}} \beta+\frac{1}{\beta}\right)-\sqrt{\left(\frac{c}{k} \theta \frac{\varepsilon_{r}}{\varepsilon_{c}} \beta\right)^{2}+2 \frac{c}{k} \theta \frac{\varepsilon_{r}}{\varepsilon_{c}}+\left(\frac{1}{\beta}-2\right)^{2}}+2 \theta}{2\left(\frac{c}{k}+\delta\right)}<s
$$

In view of the previous considerations, we can introduce the following Proposition.

Proposition 5 The steady state undergoes a Hopf bifurcation at $\varepsilon_{g}^{H}$. Such a value is included between zero and one provided (12) and (13) are satisfied. Thus, there will be a closed curve around the stationary solution which will be either stable or unstable according to the direction of the bifurcation. 
An explanation for the emergence of the Hopf is much less intuitive than the flip case and rests upon the role played by the elasticity of the recycling production function, although to reproduce the mechanism at work turns out to be quite complicate.

The analysis of the stability properties shows that under some usual conditions, steady-state may display a flip or a Hopf bifurcation. These results imply stabilization public policy. One of the central parameters, among others, is the $A$ term, which depends on the waste price $p$ and on the recovery rate $\phi(A=p \phi)$. Hence, government may use market-based instruments (tax and subsidies) to determine $p$ or command and control instruments like $\phi$, such as economic fluctuations are avoided. Actually, European governments mix these two instruments to achieve environmental objectives. For example, in the case of the packaging waste policy, since 1994, a state-registered eco-organisation subsidizes the recovery sector ${ }^{9}$ in each EU country; moreover, the 1994 EU Packaging Directive ${ }^{10}$ determines minimum recovery and recycling targets for packaging waste.

\section{Simulations}

In order to analyze the consequences of the values of the recycling parameters, we choose the following simple functional forms. Assume Cobb-Douglas production technology for the virgin sector $f(k)=F k^{s}$ and $\log$ production function for the recycling sector technology $g(R)=$ $G \ln (1+R)$ where $F$ and $G$ are scale parameters. We suppose also a log function for the households welfare, i.e. $u(c)=\ln c$. We therefore have these given values for the elasticities: $\varepsilon_{r}=1-s$ and $\varepsilon_{c}=1$. Under these parameters configuration, we compute the bifurcation values of the elasticity $\varepsilon_{g}$. Actually, we want to test the sensitivity of $\varepsilon_{g}^{F}$ and $\varepsilon_{g}^{H}$ with respect to the recovery rate $\phi$ and the waste total cost $p$. More in detail, we will plot for different realistic values of $\phi$ the values of the critical elasticity as a function of $p$. Notice that $p$ represents an economic tool chosen by the government and at the same time, it measures transaction cost paid by a State-registered eco-organisation for the resources $R$. An increase of $p$ means higher shrinkage cost and higher industrial packaging cost of waste. $p$ fluctuates for material types and also for each material of the same type. The recovery rate $\phi$ vary according to the material types; for example in 2004 in France, $\phi=55 \%$ for metals and $\phi=75 \%$ for paper and cardboards. This rate $\phi$ might vary also for each material of the same type; for example, in the case of the paper 


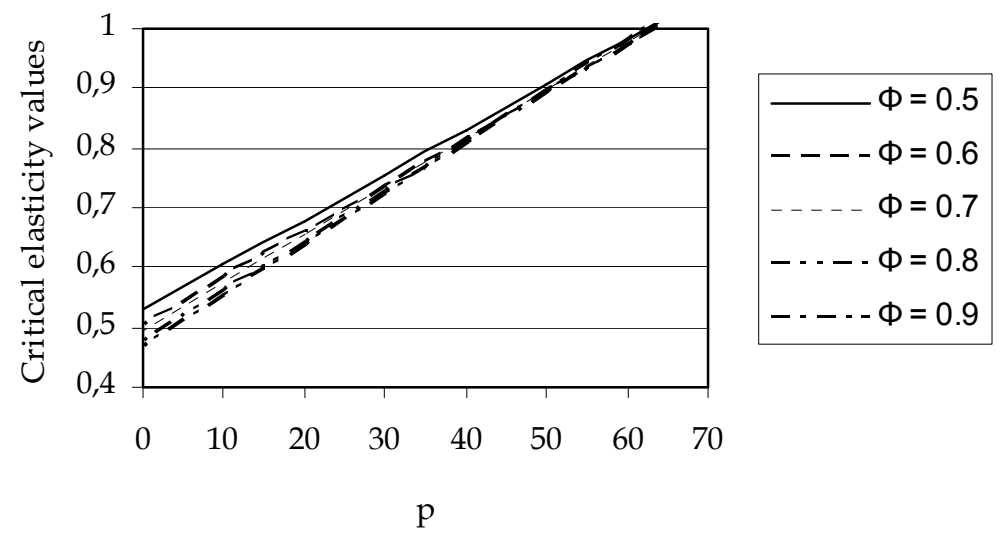

Figure 1: Flip bifurcation

and cardboards, $\phi=55 \%$ in 1997 and $\phi=75 \%$ in 2004 . In the sequel, we will retain the following structural parameters: $\beta=0.9, \delta=0.25, s=0.3, F=1, G=100$.

For the flip bifurcation, we get the following picture (see Fig. 1).

Notice that the bifurcation value of $\varepsilon_{g}^{F}$ is increasing with the waste total cost $p$ and the recovery rate $\phi$. Hence, for a given elasticity, the more the good is recyclable, the less the waste total cost is. This result seems quite realistic since the government has to fix minimum price when the good is highly recyclable. Indeed, for high recovery rate, the supply of waste that could be recycled is high too. As we can see, these values of $\varepsilon_{g}^{F}$ fall within standard intervals even when $p$ is set large enough.

Concerning the Hopf bifurcation, we have the following picture (see Fig. 2).

Again, we find the same main features: the critical elasticity is increasing with $p$ and $\phi$. However, in order to get a Hopf bifurcation the cost should be large enough. One can easily show that in correspondence with our simulations, the condition of non-negativity of profits is respected.

\section{Extensions}

\subsection{The optimal case}

It could be interesting to analyze the social planner problem of our economy. In such a case, the recycling activity is taken into account when maximizing. Actually, the social planner considers 


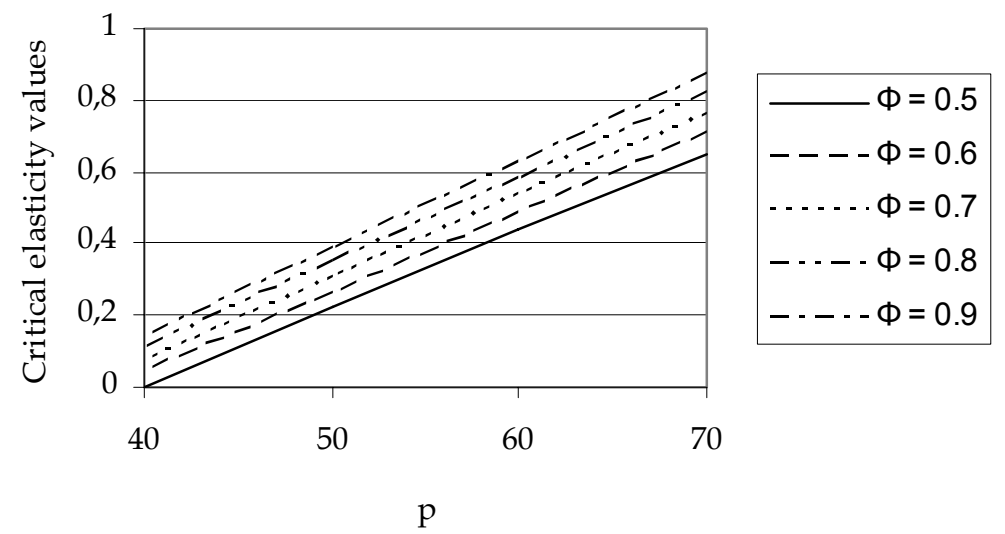

Figure 2: Hopf bifurcation

the life-cycle of investment which lasts two periods: in the first one, it is used in the standard production function while in the second, it represents the input of the recycling sector.

Straightforward computations give the following Euler equation:

$$
u^{\prime}\left(c_{t}\right)=\beta u^{\prime}\left(c_{t+1}\right)\left((1-\delta)+f^{\prime}\left(k_{t+1}\right)\right)+\beta^{2} u^{\prime}\left(c_{t+2}\right)\left(g^{\prime}\left(\phi f\left(k_{t+1}\right)\right) \phi f^{\prime}\left(k_{t+1}\right)-A f^{\prime}\left(k_{t+1}\right)\right)
$$

which together the resource constraint describes the dynamics of the model. The steady-state level of capital is obtained by solving:

$$
1=\beta\left(1-\delta+f^{\prime}(k)\right)+\beta^{2}\left(g^{\prime}(\phi f(k)) \phi f^{\prime}(k)-A f^{\prime}(k)\right)
$$

By manipulating (15), we obtain:

$$
f^{\prime}(k)=\frac{1-\beta(1-\delta)}{\beta\left(1+\beta\left(g^{\prime}(\phi f(k)) \phi-A\right)\right)}
$$

The Left Hand Side (LHS) of (16) decreases from $\infty$ to 0 , meanwhile the RHS starts from 0 and then increases monotonically by reaching $\frac{1-\beta(1-\delta)}{\beta-A}$ when $k$ becomes infinite. Notice that whatever the sign of $\beta-A$, the LHS and the RHS intersect only once. Therefore, the steady-state is unique.

The dynamics of the optimal model involves now the profit maximization activity of the recycling sector. Therefore, two regimes are possible. In the first one, profit is decreasing in $k$ i.e. $k>k_{\max }$, where $k_{\max }$ solves $k_{\max }=\arg \max g(\phi f(k)-A f(k))$, and then the social planner chooses to use only $k_{\max }$ and throws away $k-k_{\max }$. Therefore, the model boils down 
to the standard Ramsey-Cass-Koopmans one up to a constant which does not interfere with the dynamics. In the second configuration, profit are increasing in $k$ and therefore, all of the amount of capital available is used in the recycling sector.

In the latter case, the system describing the equilibrium dynamics is four dimensional and not easily tractable but could be an interesting extension to study. Obviously, some nice analytical results would disappear since the dimension of the system is higher. However, it would be possible to compute numerical solutions in this case and to compare them to the non optimal case. These results will imply to define the optimal policy for the waste public management but need also to introduce the pollution externalities generated by the waste and therefore the environmental benefits of the recycling sector. This is a topic for further research.

\subsection{Current recycling activity}

Assume no lag in the collecting and the recycling of waste by the government, i.e. the recycling sector uses the capital stock during the same period than the standard sector. This could happen for non durable goods like papers or glass. If we consider the decentralized economy, the dynamic system writes:

$$
\begin{cases}\max & \sum \beta^{t} u\left(c_{t}\right) \\ \text { w.r.t. } & c_{t}+k_{t+1}=w_{t}+\left(1-\delta+r_{t}\right) k_{t}+\pi_{t}\end{cases}
$$

and the intertemporal equilibrium is:

$$
\left\{\begin{array}{l}
u^{\prime}\left(c_{t}\right)=\beta\left(1-\delta+f^{\prime}\left(k_{t+1}\right)\right) u^{\prime}\left(c_{t+1}\right) \\
c_{t}+k_{t+1}=f\left(k_{t}\right)+(1-\delta) k_{t}+g\left(\phi f\left(k_{t}\right)\right)-A f\left(k_{t}\right) .
\end{array}\right.
$$

Notice that system (18) involves one predetermined variable, $k$ and one which is not, $c$. We can then determine the steady-state:

$$
\left\{\begin{array}{l}
f^{\prime}(k)=\frac{1}{\beta}-(1-\delta) \equiv \theta \\
c=f(k)-\delta k+g(\phi f(k))-A f(k)=-\delta k+g(\phi f(k))+(1-A) f(k)
\end{array}\right.
$$

Proposition 6 There exists a unique steady-state for the intertemporal equilibrium described by system (18).

The linearization of system (18) gives the following Jacobian matrix:

$$
J=\left[\begin{array}{cc}
Z & -1 \\
-\beta \theta \frac{c}{k} \frac{\varepsilon_{s}}{\varepsilon_{c}} Z & 1+\beta \theta \frac{c}{k} \frac{\varepsilon_{s}}{\varepsilon_{c}}
\end{array}\right]
$$

where $Z \equiv \frac{1}{\beta}+\varepsilon_{g} s \tilde{B}-A \theta$ and $\tilde{B} \equiv \delta+\frac{c}{k}+\frac{\theta}{s}(A-1)$. The Jacobian matrix is characterized by Determinant $D=Z$ and Trace $T=Z+1+\beta \theta \frac{c}{k} \frac{\varepsilon_{s}}{\varepsilon_{c}}$. From the definition of the Trace, we can 
draw the conclusion that there is no room for a saddle node bifurcation nor a Hopf bifurcation, and that the system is always determined. Conversely, there could arise a flip bifurcation when $D$ is lower than -1 . Namely, this occurs when $T=D+1+\beta \theta \frac{c}{k} \frac{\varepsilon_{s}}{\varepsilon_{c}}=-D-1$, which requires the following elasticity for the recycling production function:

$$
\varepsilon_{g}^{F}=\frac{-1-\beta \frac{\theta}{2} \frac{c}{k} \frac{\varepsilon_{s}}{\varepsilon_{c}}+A \theta-\frac{1}{\beta}}{s \delta+\frac{c}{k}+\frac{\theta}{s}(A-1)}
$$

Proposition 7 System (18) is locally determinate either a saddle or a source. In addition it undergoes a flip bifurcation when the elasticity of the recycling production function goes through $\varepsilon_{g}^{F}$ defined in (20).

\section{Conclusion}

In this paper we have analyzed an infinite horizon model in which there is a recycling sector producing a good which is perfectly substitute to the one produced by a standard constantreturns technology. The recycling production function is set to respect the parameters required by the European Directive. After a discussion on the characterization of the recycling sector, we have carried out a stability analysis. More in details, we have shown that the recycling technology may be responsible for the existence of deterministic cycles through a flip bifurcation and a closed curve surging through a Hopf-Poincaré bifurcation. These bifurcations occur for reliable parameter values and for high enough propensity to consume. These results contradict some findings, as in Boldrin and Rustichini (1994), according to which complex dynamics cannot occur in one-sector models when an inelastic labor supply is assumed. An interesting extension of the model could be that of analyzing the role played by the recycling technology in an overlapping generations framework. Indeed, in such a case, there would be an interaction between the agents' life cycle and the product' one. Another possible extension is that of including the environmental dynamics in the model.

\section{Acknowledgements}

The authors are indebted to two anonymous referees for their helpful suggestions and comments on an earlier version of this article. We are also grateful for the comments of the participants of the CREE 2007 conference and the GED seminar of CES. 


\section{Notes}

1. In France, the Decree of July 2005 concerning the disposal of electrical and electronic waste results in the application of the European Directive of 2002 (Directive WEEE 2002/96/EC). Immediately from November 2006, producers of these goods contributed funds to one of the four State-registered eco-organisations (Eco-Systèmes, ERP, Ecologic and Recyclum).

2. This is the ratio between the recovery of used papers and cardboard and the consumption of virgin papers and cardboard.

3. Source: French Confederation of the Paper, Cardboard and Cellulose Industry, 2003.

4. Source: European Federation of Glassware Containers, 2003.

5. This characteristic is a consequence of the legal definition of waste. According to the law, waste corresponds to "... all residues resulting from a process of manufacture, of transformation or of use of all substance, material, product (...) abandoned or which the producer intends to throw away...". It is therefore of no interest to the person, which renders the waste a useless or negative value. But the notion of waste cannot be precise as it depends on the technology available and on the structure of the economy: the development of the recovery and recycling sectors transforms waste into a raw material.

6. Martin (1982) conducts an in-depth analysis of how the different forms of vertical integration of the market between Alcoa, scrap recovery and recycling firms affect monopoly profit.

7. This way of waste price determination (fixed price) is an industrial reality and corresponds, for example, to the application of the 2002 EU Directive (Directive WEEE 2002/96/EC) concerning electrical and electronic material waste products, where used computers were actually recovered free of charge in 2006; it corresponds also to the guarantee of the takingback of Eco-Emballages between 1992 and 2005 in France.

8. This cost corresponds to an iceberg cost: a part of the goods disappears during transportation (Samuelson; 1954, Helpman and Krugman; 1985). 
9. The consumer pays a tax on packaged goods; this eco-contribution has to fill the gap between the re-sale price of secondary materials and the marginal cost of their recovery.

10. The 1994 Directive on Packaging and Packaging Waste (94/62/EC) and the 2004 Packaging Directive Amendments (2004/12/EC).

\section{References}

Boldrin, M. and A. Rustichini (1994) Growth and Indeterminacy in Dynamic Models with Externalities. Econometrica 62, 323-343.

Choe, C. and I. M. Fraser (1999) An economic analysis of household waste management. Journal of Environmental Economics and Management 38, 234-246.

Dasgupta, P.S. and G. Heal (1979) Economic Theory of Exhaustible Resources. James NibsetCambridge University Press: Cambridge.

Fullerton, D. and T. C. Kinnaman (1995) Garbage, Recycling, and Illicit Burning or Dumping. Journal of Environmental Economics and Management 29, 78-91.

Fullerton, D. and W. Wu (1998) Policies for Green Design. Journal of Environmental Economics and Management 36, 131-148.

Gaskins, D. W. (1974) Alcoa Revisited: Welfare Implications of a Secondhand Market. Journal of Economic Theory 7, 254-271.

Grandmont, J.M. (1988) Non linear Difference Equations, Bifurcations and Chaos: an Introduction. CEPREMAP DP-8811.

Grant, D. (1999) Recycling and market power: A more general model and re-evaluation of the evidence. International Journal of Industrial Organization 17, 59-80.

Helpman, E. and P. R. Krugman (1985) Market Structure and Foreign Trade. The MIT Press: Cambridge.

Keeler, A. G. and M. Renkow (1994) Haul Trash or Haul Ash: Energy Recovery as a Component of Local Solid Waste Management. Journal of Environmental Economics and Management 27, 205-217.

Lusky, R. (1976) A model of recycling and pollution control. Canadian Journal of Economics 9, 91-101.

Mäler, K. G. (1974) Environmental Economics: A Theoretical Inquiry. John Hopkins University Press: Baltimore. 
Martin, R. E. (1982) Monopoly Power and the Recycling of Raw Materials. The Journal of Industrial Economics 30, 104-419.

Palmer, K. and M. Walls (1997) Optimal policies for solid waste disposal: Taxes, subsidies and standards. Journal of Public Economics 65, 193-205.

Samuelson, P. A. (1954) The transfer problem and transport cost, II: analysis of effects of trade impediments. Economic Journal 64, 264-289.

Seegmuller, T. and A. Verchère (2007) A note on indeterminacy in overlapping generations economies with environment and endogenous labor supply. Macroeconomic Dynamics 11, 423429.

Suslow, V. Y. (1986) Estimating monopoly behavior with competitive recycling: an application to Alcoa. Rand Journal of Economics 17, 389-403.

Swan, P. L. (1980) Alcoa: The Influence of Recycling on Monopoly Power. Journal of Political Economy 88, 76-99.

\section{Appendix}

\section{The Hopf bifurcation}

If we develop (10) and using (9), by identification we obtain:

$$
\left\{\begin{array}{l}
a=\frac{T-D}{2} \\
a=\frac{\Sigma-1}{2 D}
\end{array}\right.
$$

In order to find an $a$ which verifies both equalities, we exploit the expression of our polynomial which is

$$
P(\lambda)=\lambda^{3}-\left(1+\frac{1}{\beta}+\frac{c}{k} \theta \frac{\varepsilon_{r}}{\varepsilon_{c}} \beta\right) \lambda^{2}+\left(\frac{1}{\beta}-B\right) \lambda+B
$$

Again by identification, we have

$$
\left\{\begin{array}{l}
T=\left(1+\frac{1}{\beta}+\frac{c}{k} \theta \frac{\varepsilon_{r}}{\varepsilon_{c}} \beta\right) \\
\Sigma=\left(\frac{1}{\beta}-B\right) \\
D=(-B)
\end{array}\right.
$$

Hence, using (21), we obtain a polynomial of second degree in $B$ such that $B^{2}+B\left(\frac{c}{k} \theta \frac{\varepsilon_{r}}{\varepsilon_{c}} \beta+\frac{1}{\beta}\right)+$ $\frac{1}{\beta}-1=0$. In order to get two real roots $B_{1,2}$, the discriminant must be positive or zero. The discriminant $\Delta$ of such a polynomial is

$$
\Delta=\left(\frac{c}{k} \theta \frac{\varepsilon_{r}}{\varepsilon_{c}} \beta\right)^{2}+2 \frac{c}{k} \theta \frac{\varepsilon_{r}}{\varepsilon_{c}}+\left(\frac{1}{\beta}-2\right)^{2}>0
$$


The solutions of the polynomial are therefore:

$$
\begin{aligned}
& B_{1}=\frac{-\left(\frac{c}{k} \theta \frac{\varepsilon_{r}}{\varepsilon_{c}} \beta+\frac{1}{\beta}\right)+\sqrt{\left(\frac{c}{k} \theta \frac{\varepsilon_{r}}{\varepsilon_{c}} \beta\right)^{2}+2 \frac{c}{k} \theta \frac{\varepsilon_{r}}{\varepsilon_{c}}+\left(\frac{1}{\beta}-2\right)^{2}}}{2} \\
& B_{2}=\frac{-\left(\frac{c}{k} \theta \frac{\varepsilon_{r}}{\varepsilon_{c}} \beta+\frac{1}{\beta}\right)-\sqrt{\left(\frac{c}{k} \theta \frac{\varepsilon_{r}}{\varepsilon_{c}} \beta\right)^{2}+2 \frac{c}{k} \theta \frac{\varepsilon_{r}}{\varepsilon_{c}}+\left(\frac{1}{\beta}-2\right)^{2}}}{2}
\end{aligned}
$$

Notice now that the term $a=\frac{T-D}{2}$ is such that either

$$
a_{1}=\frac{\left(1+\frac{1}{\beta}+\frac{c}{k} \theta \frac{\varepsilon_{r}}{\varepsilon_{c}} \beta\right)+\frac{-\left(\frac{c}{k} \theta \frac{\varepsilon_{r}}{\varepsilon_{c}} \beta+\frac{1}{\beta}\right)+\sqrt{\left(\frac{c}{k} \theta \theta \frac{\varepsilon_{r}}{\varepsilon_{c}} \beta\right)^{2}+2 \frac{c}{k} \theta \frac{\varepsilon_{r}}{\varepsilon_{c}}+\left(\frac{1}{\beta}-2\right)^{2}}}{2}}{2}
$$

or

$$
a_{2}=\frac{\left(1+\frac{1}{\beta}+\frac{c}{k} \theta \theta \frac{\varepsilon_{r}}{\varepsilon_{c}} \beta\right)+\frac{-\left(\frac{c}{k} \theta \frac{\varepsilon_{r}}{\varepsilon_{c}} \beta+\frac{1}{\beta}\right)-\sqrt{\left(\frac{c}{k} \theta \frac{\varepsilon_{r}}{\varepsilon_{c}} \beta\right)^{2}+2 \frac{c}{k} \theta \frac{\varepsilon_{r}}{\varepsilon_{c}}+\left(\frac{1}{\beta}-2\right)^{2}}}{2}}{2}
$$

If we want now $|a|<1$, it must be

$$
\left|2+\frac{1}{\beta}+\frac{c}{k} \theta \frac{\varepsilon_{r}}{\varepsilon_{c}} \beta \pm \sqrt{\left(\frac{c}{k} \theta \frac{\varepsilon_{r}}{\varepsilon_{c}} \beta\right)^{2}+2 \frac{c}{k} \theta \frac{\varepsilon_{r}}{\varepsilon_{c}}+\left(\frac{1}{\beta}-2\right)^{2}}\right|<4
$$

If the solution is $a_{1}$, since all the terms are positive, we have

$$
2+\frac{1}{\beta}+\frac{c}{k} \theta \frac{\varepsilon_{r}}{\varepsilon_{c}} \beta+\sqrt{\left(\frac{c}{k} \theta \frac{\varepsilon_{r}}{\varepsilon_{c}} \beta\right)^{2}+2 \frac{c}{k} \theta \frac{\varepsilon_{r}}{\varepsilon_{c}}+\left(\frac{1}{\beta}-2\right)^{2}}<4
$$

which implies the contradiction $0<-4 \frac{c}{k} \theta \frac{\varepsilon_{r}}{\varepsilon_{c}} \beta$. In the case instead in which the solution is $a_{2}=$ $2+\frac{1}{\beta}+\frac{c}{k} \theta \frac{\varepsilon_{r}}{\varepsilon_{c}} \beta-\sqrt{\left(\frac{c}{k} \theta \frac{\varepsilon_{r}}{\varepsilon_{c}} \beta\right)^{2}+2 \frac{c}{k} \theta \frac{\varepsilon_{r}}{\varepsilon_{c}}+\left(\frac{1}{\beta}-2\right)^{2}}$, the result can be either positive or negative. In the case where $a_{2}$ is negative we have $-2-\frac{1}{\beta}-\frac{c}{k} \theta \frac{\varepsilon_{r}}{\varepsilon_{c}} \beta+\sqrt{\left(\frac{c}{k} \theta \frac{\varepsilon_{r}}{\varepsilon_{c}} \beta\right)^{2}+2 \frac{c}{k} \theta \frac{\varepsilon_{r}}{\varepsilon_{c}}+\left(\frac{1}{\beta}-2\right)^{2}}<4$ and therefore we obtain that the inequality $-2\left(\frac{1}{\beta}+2\right)<3 \frac{c}{k} \theta \frac{\varepsilon_{r}}{\varepsilon_{c}} \beta$ is always verified; thus $\left|a_{2}\right|<1$. In the case in which $a_{2}$ is positive we have $2+\frac{1}{\beta}+\frac{c}{k} \theta \frac{\varepsilon_{r}}{\varepsilon_{c}} \beta-\sqrt{\left(\frac{c}{k} \theta \frac{\varepsilon_{r}}{\varepsilon_{c}} \beta\right)^{2}+2 \frac{c}{k} \theta \frac{\varepsilon_{r}}{\varepsilon_{c}}+\left(\frac{1}{\beta}-2\right)^{2}}<$ 4 which implies $0>-2 \beta$, that is always satisfied. Therefore the system undergoes a Hopf bifurcation when

$$
B=B_{2}=\frac{-\left(\frac{c}{k} \theta \frac{\varepsilon_{r}}{\varepsilon_{c}} \beta+\frac{1}{\beta}\right)-\sqrt{\left(\frac{c}{k} \theta \frac{\varepsilon_{r}}{\varepsilon_{c}} \beta\right)^{2}+2 \frac{c}{k} \theta \frac{\varepsilon_{r}}{\varepsilon_{c}}+\left(\frac{1}{\beta}-2\right)^{2}}}{2}
$$


since in such a case there exists $a_{2}$ with $|a|<1$. We need eventually to prove that condition (24) is satisfied for some parameters configuration. Since we have

$$
B \equiv \varepsilon_{g} s\left(\frac{c}{k}+\delta+(A-1) \frac{\theta}{s}\right)-A \theta
$$

it must be

$$
\varepsilon_{g} s\left(\frac{c}{k}+\delta+(A-1) \frac{\theta}{s}\right)-A \theta=\frac{-\left(\frac{c}{k} \theta \frac{\varepsilon_{r}}{\varepsilon_{c}} \beta+\frac{1}{\beta}\right)-\sqrt{\left(\frac{c}{k} \theta \frac{\varepsilon_{r}}{\varepsilon_{c}} \beta\right)^{2}+2 \frac{c}{k} \theta \frac{\varepsilon_{r}}{\varepsilon_{c}}+\left(\frac{1}{\beta}-2\right)^{2}}}{2}
$$

which is satisfied for every $\varepsilon_{g}$ provided that $A$ is chosen appropriately (since $B=B_{2}<0, A$ must be large enough).

Therefore, after tedious computations, we find that the Hopf bifurcation value for $\varepsilon_{g}$ is given by eq. (11) . 